\begin{tabular}{|c|c|}
\hline Title & Synthesis, structure, and magnetic and dielectric properties of magnetoelectric $\mathrm{BaDyFeO} 4$ ferrite \\
\hline Author(s) & $\begin{array}{l}\text { Belik, A lexei A.; T erada, Noriki; Katsuya, Y oshio; T anaka, Masahiko; Glazkova, Iana S.; Sobolev, A lexey V.; } \\
\text { Presniakov, Igor A.; Y amaura, Kazunari }\end{array}$ \\
\hline Citation & $\begin{array}{l}\text { Journal of alloys and compounds, 811, UNSP } 151963 \\
\text { https://doi.org/10.1016 } / \text {.jall com.2019.151963 }\end{array}$ \\
\hline Issue Date & 2019-11-30 \\
\hline Doc URL & http:/hdl.handle.net/2115/83352 \\
\hline Rights & https://www.el sevier.com \ournal s/ ournal- of-all oys and-compounds/0925-8388/guide for-author\$13300 \\
\hline Rights(URL) & https://reativecommons.org/icenses/by-nc-nd/4.0/ \\
\hline Type & article \\
\hline File Information & BaDyFe0 4.pdf \\
\hline
\end{tabular}

Instructions for use 


\title{
Synthesis, structure, and magnetic and dielectric properties of magnetoelectric $\mathrm{BaDyFeO}_{4}$ ferrite
}

\author{
Alexei A. Belik ${ }^{\text {a, }}{ }^{*}$, Noriki Terada ${ }^{\text {b }}$, Yoshio Katsuya ${ }^{\text {c }}$, Masahiko Tanaka ${ }^{\text {c, }}$ \\ Iana S. Glazkova ${ }^{\text {d, Alexey V. Sobolev }}{ }^{\text {d, Igor A. Presniakov }}{ }^{\text {d }}$, Kazunari Yamaura ${ }^{\text {a e }}$
}

${ }^{a}$ Research Center for Functional Materials, National Institute for Materials Science (NIMS), Namiki 1-1, Tsukuba, Ibaraki 305-0044, Japan

b National Institute for Materials Science (NIMS), Sengen 1-2-1, Tsukuba, Ibaraki 305-0047, Japan

c Synchrotron X-ray Station at SPring-8, NIMS, Kouto 1-1-1, Sayo-cho, Hyogo 679-5148, Japan

d Department of Chemistry, Lomonosov Moscow State University, Leninskie Gory, 119992 Moscow, Russia.

e Graduate School of Chemical Sciences and Engineering, Hokkaido University, North 10 West 8, Kita-ku, Sapporo, Hokkaido 060-0810, Japan 


\begin{abstract}
$\mathrm{BaDyFeO}_{4}$ was prepared by a conventional solid-state method in air at $1573 \mathrm{~K}$. It crystallizes in space group Pnma (No. 62) with $a=13.16861(1) \AA, b=5.70950(1) \AA$, and $c=$ 10.26783(1), and it is isostructural with $\mathrm{BaYFeO}_{4}$. Three magnetic transitions were found in $\mathrm{BaDyFeO}_{4}$ at $T_{\mathrm{N} 3}=9 \mathrm{~K}, T_{\mathrm{N} 2}=23 \mathrm{~K}$, and $T_{\mathrm{N} 1}=47 \mathrm{~K}$ in zero magnetic field in comparison with two magnetic transitions observed in $\mathrm{BaYFeO}_{4}$. Magnetic-field-induced transitions were also detected in $\mathrm{BaDyFeO}_{4}$ at 18 and $28 \mathrm{kOe}$ (at $T=1.8 \mathrm{~K}$ ). Frequency-dependent broad dielectric peaks were observed in $\mathrm{BaDyFeO}_{4}$ spanning between $T_{\mathrm{N} 2}$ and $T_{\mathrm{N} 1}$ and centred at $35 \mathrm{~K}$ - this temperature does not coincide with any $T_{\mathrm{N}}$. No dielectric anomalies were found at $T_{\mathrm{N} 1}$ and $T_{\mathrm{N} 3}$, while very weak frequency-independent dielectric anomalies were detected at $T_{\mathrm{N} 2}$. Positive and negative magnetodielectric effects were measured in $\mathrm{BaDyFeO}_{4}$ (within a range of -0.8 and $+0.4 \%$ up to $90 \mathrm{kOe}$ ) reflecting magnetic-field dependence of dielectric constant. Pyroelectric current measurements did not detect any ferroelectricity in $\mathrm{BaDyFeO}_{4}$ under measurement conditions used. No dielectric anomalies and no magnetodielectric effects were found in $\mathrm{BaYFeO}_{4}$.
\end{abstract}

Keywords: ferrites; magnetodielectric effect; multiferroics; crystal structure 


\section{Introduction}

A phenomenon of spin-induced ferroelectricity has recently received a lot of attention [13]. It usually takes place when the symmetry of a long-range magnetic structure is polar. In more general words, it requires non-trivial, complex magnetic structures, for example, sinusoidal-type and cycloid-type orderings [3]. Non-trivial magnetic structures could be realized (1) in systems with very complex networks of physical connections among magnetic ions and (2) in systems with simple networks of physical connections among magnetic ions (for example, in perovskites with three-dimensional connections of corner-shared octahedra) but with the presence of spin frustration or complex networks of spin exchange interactions [1-3]. Spin-induced ferroelectricity has been discovered in many previously known magnetic materials.

The exploration of new materials with complex connection networks of magnetic ions is a way to expand the family of spin-induced multiferroics. A new barium yttrium ferrite, $\mathrm{BaYFeO}_{4}$, was recently reported with a complex magnetic sublattice [4, 5]. In $\mathrm{BaYFeO}_{4}$, there are $\left[\mathrm{Fe}_{4} \mathrm{O}_{28}\right]$ clusters or rings formed by corner-shared $\mathrm{FeO}_{5}$ square pyramids and $\mathrm{FeO}_{6}$ octahedra; the rings, in turn, share corners to form one-dimensional chains along the $b$ axis (Figure 1b). $\mathrm{BaYFeO}_{4}$ is isostructural with $\mathrm{Ba}_{2} \mathrm{CuPtY}_{2} \mathrm{O}_{8}$ and related compounds [6]. Neutron powder diffraction and magnetic susceptibility measurements showed the existence of two magnetic transitions in $\mathrm{BaYFeO}_{4}$ with $T_{\mathrm{N} 2}=33 \mathrm{~K}$ and $T_{\mathrm{N} 1}=48 \mathrm{~K}[4,5]$. Below $T_{\mathrm{N} 1}$, a spin-density wave structure was suggested, and below $T_{\mathrm{N} 2}$ - an incommensurate cycloid structure [5, 7]. Very weak spin-induced ferroelectricity was found below $T_{\mathrm{N} 2}$ [8]. Spinglass-like behavior and relaxation of magnetization below $17 \mathrm{~K}$ were reported in Ref. 9. There are also some puzzling behaviours of $\mathrm{BaYFeO}_{4}$, for example, (1) the absence of any specific heat anomalies at $T_{\mathrm{N} 1}$ and $T_{\mathrm{N} 2}$ despite a large spin of $\mathrm{Fe}^{3+}(S=5 / 2)$ indicating a very small entropy release at the magnetic transition temperatures [4] and (2) the absence of Curie-Weiss behavior, reported in all papers [4, 5, 8, 9], up to $650 \mathrm{~K}$ [5].

Ferrites show rich crystal chemistry and magnetism [10]. There are many ferrites with different compositions in Ba-R-Fe-O systems, where $\mathrm{R}$ is a rare-earth cation and $\mathrm{Y}$ [11], for 
example, $\mathrm{YBaFe}_{4} \mathrm{O}_{7}, \mathrm{Ba}_{3} \mathrm{YFe}_{2} \mathrm{O}_{7.5}, \mathrm{YBa}_{2} \mathrm{Fe}_{3} \mathrm{O}_{8}, \mathrm{YBa}_{2} \mathrm{FeO}_{5.5}$, and $\mathrm{YBaFe}_{2} \mathrm{O}_{5}$. In Ba-Dy-Fe-O system, $\mathrm{DyBaFe}_{4} \mathrm{O}_{7}$ [10], $\mathrm{DyBaFe}_{2} \mathrm{O}_{5}$ [12], and $\mathrm{DyBa}_{2} \mathrm{Fe}_{3} \mathrm{O}_{8}$ [13] have been reported. Ferrites with such a simple composition, $\mathrm{BaRFeO}_{4}$, are reported only for $\mathrm{R}=\mathrm{La}$ and $\mathrm{Y}$, and $\mathrm{BaLaFeO}_{4}$ crystallizes in a different crystal structure [14]. This fact suggests that at least two different structure types are realized in $\mathrm{BaRFeO}_{4}$ depending on the size of rare-earth elements, and $\mathrm{BaRFeO}_{4}$ ferrites can exist for other rare-earth elements.

Therefore, in the present work, we extended the family of $\mathrm{BaRFeO}_{4}$ simple ferrites and report on the synthesis, crystal structure, and properties of $\mathrm{BaDyFeO}_{4}$. We selected $\mathrm{Dy}^{3+}$ as it has the close ionic radius with that of $\mathrm{Y}^{3+}$. In addition, $\mathrm{Dy}^{3+}$ has the largest magnetic moment among rare-earth cations, and the appearance of an additional magnetic sublattice could significantly modify properties of $\mathrm{BaRFeO}_{4}$ ferrites. We also report on some properties of $\mathrm{BaYFeO}_{4}$. First, in order to understand effects of the $\mathrm{Dy}^{3+}$ sublattice in $\mathrm{BaDyFeO}_{4}$ it is necessary to make comparison between $\mathrm{BaDyFeO}_{4}$ and $\mathrm{BaYFeO}_{4}$, but some properties of $\mathrm{BaYFeO}_{4}$ have not been reported yet, such as, magnetic-field dependence of specific heat and magnetodielectric effects. Second, more experimental information is needed to understand intrinsic properties of $\mathrm{BaYFeO}_{4}$, especially the influence of synthesis conditions and routes and the sample quality on properties. We found that $\mathrm{BaDyFeO}_{4}$ shows a more complex behavior than that of $\mathrm{BaYFeO}_{4}$ from the viewpoint of magnetism and lowtemperature dielectric properties. We observed positive and negative magnetodielectric effects in $\mathrm{BaDyFeO}_{4}$ due to magnetic-field dependence of dielectric constant, but no magnetodielectric effects in $\mathrm{BaYFeO}_{4}$. No signs of spin-induced ferroelectricity were detected in $\mathrm{BaDyFeO}_{4}$ within measurement conditions used and the sensitivity of our methods.

\section{Experimental}

$\mathrm{BaDyFeO}_{4}$ and $\mathrm{BaYFeO}_{4}$ were prepared from stoichiometric mixtures of $\mathrm{BaCO}_{3}, \mathrm{Y}_{2} \mathrm{O}_{3}$, $\mathrm{Dy}_{2} \mathrm{O}_{3}$, and $\mathrm{Fe}_{2} \mathrm{O}_{3}$, where we used about $25 \%$ of $\mathrm{Fe}_{2} \mathrm{O}_{3}$ enriched by ${ }^{57} \mathrm{Fe}$ (95.5 \%) in case of $\mathrm{BaDyFeO}_{4}$ (for future Mössbauer studies). $\mathrm{Y}_{2} \mathrm{O}_{3}$ and $\mathrm{Dy}_{2} \mathrm{O}_{3}$ were dried at $1270 \mathrm{~K}$ before use, $\mathrm{Fe}_{2} \mathrm{O}_{3}$ - at $1170 \mathrm{~K}$, and $\mathrm{BaCO}_{3}$ - at $870 \mathrm{~K}$. The mixtures were pressed into pellets and 
annealed in air on Pt foil at $1430 \mathrm{~K}$ for $48 \mathrm{~h}, 1520 \mathrm{~K}$ for $18 \mathrm{~h}$, and $1570 \mathrm{~K}$ for $38 \mathrm{~h}$ in case of $\mathrm{BaDyFeO}_{4}$ and at $1430 \mathrm{~K}$ for $48 \mathrm{~h}$ and $1520 \mathrm{~K}$ for $48 \mathrm{~h}$ in case of $\mathrm{BaYFeO}_{4}$ (with grinding after each step). After such procedures, $\mathrm{BaDyFeO}_{4}$ contained about 2 weight \% of $\mathrm{DyFeO}_{3}$ impurity; however, $\mathrm{BaYFeO}_{4}$ contained about 7.7 wt. \% of $\mathrm{BaFeO}_{3}$ and 2.2 wt. \% of $\mathrm{Y}_{2} \mathrm{O}_{3}$ impurities. Therefore, $\mathrm{BaYFeO}_{4}$ was additionally annealed at $6 \mathrm{GPa}$ and about $1420 \mathrm{~K}$ for 90 min in an Au capsule using a belt-type high-pressure machine (heating time to the synthesis temperature was $10 \mathrm{~min}$ ). After the high-pressure heat treatment, the sample was quenched to room temperature (RT), and the pressure was slowly released. After the high-pressure treatment, $\mathrm{BaYFeO}_{4}$ contained about 2.0 wt. \% of $\mathrm{YFeO}_{3}$ impurity. We note that additional annealing of $\mathrm{BaYFeO}_{4}$ at $1570 \mathrm{~K}$ for $48 \mathrm{~h}$ in air (after annealing at $1430 \mathrm{~K}$ for $48 \mathrm{~h}$ and at $1520 \mathrm{~K}$ for $48 \mathrm{~h}$ in air, the sample was split into two batches: the first one was for the HP treatment, the second one - for the additional annealing in air) did not significantly improve the sample quality in our case as it contained about 3.9 wt. \% of $\mathrm{BaFeO}_{3}, 0.6$ wt. \% of $\mathrm{Y}_{2} \mathrm{O}_{3}$, and 1.4 wt. \% of $\mathrm{YFeO}_{3}$ impurities.

X-ray powder diffraction (XRPD) data were collected at RT on a RIGAKU MiniFlex600 diffractometer using $\mathrm{CuK} \alpha$ radiation (2 $\theta$ range of $8-110^{\circ}$, a step width of $0.02^{\circ}$, and a scan speed of $\left.1 \mathrm{deg} / \mathrm{min}\right)$. XRPD data were analysed by the Rietveld method using RIETAN-2000 [15]. Weight fractions of impurities were estimated from refined scale factors by RIETAN-2000 after the Rietveld analysis [15].

Synchrotron XRPD data of $\mathrm{BaDyFeO}_{4}$ were measured at RT on a large Debye-Scherrer camera at the undulator beamline BL15XU of SPring-8 $[16,17]$. The intensity data were collected between $3^{\circ}$ and $71.40^{\circ}$ at $0.003^{\circ}$ intervals in $2 \theta$; the incident beam was monochromatized at $\lambda=0.65298 \AA$. The sample was packed into a Lindemann glass capillary (inner diameter: $0.1 \mathrm{~mm}$ ), which was rotated during the measurement. The absorption coefficient was also measured. The Rietveld analysis was performed using the RIETAN-2000 program [15].

Magnetic measurements were performed on SQUID magnetometers (Quantum Design, MPMS-1T and MPMS-XL-7T) between 2 and $350 \mathrm{~K}$ (or $400 \mathrm{~K}$ ) in different applied fields under both zero-field-cooled (ZFC) and field-cooled on cooling (FCC) conditions. Isothermal magnetization measurements were performed between -70 and $70 \mathrm{kOe}$ at 
different temperatures. Specific heat, $C_{\mathrm{p}}$, at different magnetic fields (0-90 kOe) was recorded between 2 and $300 \mathrm{~K}$ on cooling and heating by a pulse relaxation method using a commercial calorimeter (Quantum Design PPMS). Dielectric properties were measured using a NOVOCONTROL Alpha-A High Performance Frequency Analyzer between 3 and $300 \mathrm{~K}$ on cooling and heating in the frequency range of $100 \mathrm{~Hz}$ and $2 \mathrm{MHz}$ and at different magnetic fields; both magnetic and electric fields were perpendicular to the flat surfaces of pellets. Pyroelectric current measurements were done with a Keithley 6517B electrometer. Poling in different positive and negative electric fields was performed on cooling from $100 \mathrm{~K}$ to $2 \mathrm{~K}$ at different magnetic fields; at $2 \mathrm{~K}$, the electric field was removed and electrodes were shorted. Measurements (with a heating rate of $8.1 \mathrm{~K} / \mathrm{min}$ for $\mathrm{BaDyFeO}_{4}$ and $4.3 \mathrm{~K} / \mathrm{min}$ for $\mathrm{BaYFeO}_{4}$ ) were started after the background current was below 1 pA for more than 5 min. In case of $\mathrm{BaDyFeO}_{4}$, bias electric field measurements were also used, when the sample was cooled to $2 \mathrm{~K}$ under a zero electric field; at $2 \mathrm{~K}$, an electric field of $390 \mathrm{kV} / \mathrm{m}$ was applied; and current was measured on heating with a rate of $8.1 \mathrm{~K} / \mathrm{min}$. Temperature and magnetic fields were controlled by a PPMS. Pieces of pellets were used in all magnetic, specific heat, dielectric, and pyroelectric current measurements.

Time-dependent magnetization relaxation was measured on MPMS 1T under the following protocol: a sample was cooled from $100 \mathrm{~K}$ to a measurement temperature in a nominal zero magnetic field (after a reset magnet procedure). At the measurement temperature, a magnetic field of 1 kOe was applied (in the no-overshoot mode), and magnetization was measured as a function of time. In Ref [9], a different protocol was used, when a sample was cooled under $1 \mathrm{kOe}$, and magnetization was measured under nominal zero magnetic field. However, we did not use this protocol because there are always trapped fields inside magnetometers. Values and signs of trapped fields depend on a magnetometer, magnetic-field history, and how the zero field was set (there are oscillate and no-overshoot modes on Quantum Design MPMS). These features deteriorate the reproducibility. Moreover, magnetization should be zero under real zero magnetic field in pure antiferromagnets, and any measurable magnetization will originate from non-zero real magnetic field or extrinsic contributions. 


\section{Results and Discussion}

$\mathrm{BaDyFeO}_{4}$ was found to be isostructural with $\mathrm{BaYFeO}_{4}$. Therefore, we used structural data of $\mathrm{BaYFeO}_{4}$ [4] as the initial model for the crystal structure refinement of $\mathrm{BaDyFeO}_{4}$ by the Rietveld method. The crystal structure parameters of $\mathrm{BaDyFeO}_{4}$ are summarized in Table 1. Primary bond lengths and bond-valence sums (BVS) [18] and given in Table 2. Figure 2 shows the fitting results with experimental, calculated, and difference synchrotron XRPD patterns. The BVS values of $+2.1\left(\right.$ for $\mathrm{Ba}^{2+}$ ), $+3.1\left(\right.$ for $\mathrm{Dy}^{3+}$ ), and $+2.8\left(\right.$ for $\mathrm{Fe}^{3+}$ ) are close to the expected values. The crystal structure of $\mathrm{BaDyFeO}_{4}$ is illustrated on Figure 1. The iron sublattice was described in the introduction. There is a Dy magnetic sublattice in $\mathrm{BaDyFeO}_{4}$,

illustrated on Figures 1c and 1d, in comparison with $\mathrm{BaYFeO}_{4}$. The Dy1 and Dy2 sites have seven-fold oxygen coordination environments. The $\mathrm{Dy}_{10} \mathrm{O}_{7}$ and $\mathrm{Dy} 2 \mathrm{O}_{7}$ polyhedra are joined by edges forming one-dimensional chains along the $b$ axis. While chains of the $\mathrm{Fe}_{5} \mathrm{O}_{5}$ and $\mathrm{Fe}_{6} \mathrm{O}_{6}$ polyhedra are isolated from each other (Figure 1a), there are corner-shared connections between chains of the $\mathrm{Dy}_{10} \mathrm{O}_{7}$ and $\mathrm{Dy}_{2} \mathrm{O}_{7}$ polyhedra (Figure 1c).

Magnetic susceptibilities of $\mathrm{BaYFeO}_{4}$ and $\mathrm{BaDyFeO}_{4}$ are shown on Figure 3. Two magnetic transitions were observed in $\mathrm{BaYFeO}_{4}$ at $T_{\mathrm{N} 1}=47 \mathrm{~K}$ and $T_{\mathrm{N} 2}=35 \mathrm{~K}$ at magnetic fields from 100 Oe to $70 \mathrm{kOe}$. The transitions could be more clearly seen on the $d(\chi T) / d T$ versus $T$ curves (the inset of Figure 3a). No Curie-Weiss behavior was observed in $\mathrm{BaYFeO}_{4}$, also in agreement with previous reports, and magnetic susceptibilities were fielddependent up to $400 \mathrm{~K}$. This behavior is a strong indication of the presence of a ferromagnetic-like impurity. We could clearly detect $\mathrm{YFeO}_{3}$ impurity in our sample by XRPD. $\mathrm{YFeO}_{3}$ has strong ferromagnetic-like properties (due to canting of antiferromagnetically aligned spins), and it has $T_{\mathrm{N}} \approx 655 \mathrm{~K}$ [19]. Traces of $\mathrm{YFeO}_{3}$ impurity (or other magnetic impurities) in the previous reports could be a reason for the absence of the Curie-Weiss behavior up to $650 \mathrm{~K}$ [5], and the absence of the Curie-Weiss behavior could serve as an indicator of the presence of impurities. The Néel temperatures of $\mathrm{BaYFeO}_{4}$ found in our work $\left(T_{\mathrm{N} 1}=47 \mathrm{~K}\right.$ and $\left.T_{\mathrm{N} 2}=35 \mathrm{~K}\right)$ are in very good agreement with previous reports $[4,8,9]$. However, the shape of $\chi$ versus $T$ curves was very different in different reports. 
This fact gives strong evidence that the reported $\chi$ versus $T$ curves of $\mathrm{BaYFeO}_{4}$ are sampledependent (or more precisely, strongly impurity-dependent), and the intrinsic behavior has yet to be determined.

Five anomalies could be seen on the $d(\chi T) / d T$ versus $T$ curves of $\mathrm{BaDyFeO}_{4}$ (the inset of Figure 3b). No difference was observed between ZFC and FCC curves above 1 kOe; therefore, only one of the two curves is shown (Figure 3). The anomalies near $59 \mathrm{~K}$ (and a small difference between the ZFC and FCC curves at $100 \mathrm{Oe}$ ) could originate from a $\mathrm{Fe}^{3+}$ spin reorientation transition in $\mathrm{DyFeO}_{3}$ impurity, and the anomalies at $4 \mathrm{~K}$ - from a $\mathrm{Dy}^{3+}$ ordering transition in $\mathrm{DyFeO}_{3}$ impurity [20]. The anomalies near $59 \mathrm{~K}$ disappeared above 1 $\mathrm{kOe}$ in agreement with strong suppression of the spin reorientation transition in $\mathrm{DyFeO}_{3}$ by magnetic fields [21]. Other anomalies at 9, 23, and $47 \mathrm{~K}$ should be related to magnetic transitions in the main $\mathrm{BaDyFeO}_{4}$ phase. The transitions at $T_{\mathrm{N} 1}=47 \mathrm{~K}$ and $T_{\mathrm{N} 2}=23 \mathrm{~K}$ could originate from orderings of the $\mathrm{Fe}^{3+}$ sublattice. The transition at $T_{\mathrm{N} 3}=9 \mathrm{~K}$ is probably due to ordering of the $\mathrm{Dy}^{3+}$ sublattice. A noticeable field-dependence was present in the paramagnetic temperature range of $\mathrm{BaDyFeO}_{4}$ up to $400 \mathrm{~K}$ (Figure S1) similar to $\mathrm{BaYFeO}_{4}$; this behavior could be caused by the presence of $\mathrm{DyFeO}_{3}$ impurity. Nevertheless, the CurieWeiss behavior was observed in the paramagnetic temperature range with the estimated effective magnetic moment of $11.28 \mu_{\mathrm{B}} /$ f.u. and the Curie-Weiss temperature of $-34 \mathrm{~K}$ (we used an FCC $\chi^{-1}$ versus $T$ curve measured at $70 \mathrm{kOe}$ from 395 to $250 \mathrm{~K}$ for fitting), where the calculated effective magnetic moment of $\mathrm{BaDyFeO}_{4}$ is $12.14 \mu_{\mathrm{B}} /$ f.u. (Figure S1a). The observation of the Curie-Weiss behavior is probably caused by a large moment of $\mathrm{Dy}^{3+}$ cations, which dominates over $\mathrm{DyFeO}_{3}$ impurity at high magnetic fields. $\chi$ versus $T$ curves of $\mathrm{BaDyFeO}_{4}$ strongly depended on the measurement magnetic field (Figure 4a). Anomalies on the $d(\chi T) / d T$ versus $T$ curves remained the same at $T_{\mathrm{N} 3}$ up to about $18 \mathrm{kOe}$; they were gradually smeared at $T_{\mathrm{N} 1}$ with the increase of the measurement magnetic field; on the other hand, the anomalies showed strong field-dependence at $T_{\mathrm{N} 2}$ with sharp peaks at $23 \mathrm{~K}$ at 1 kOe, $20 \mathrm{~K}$ at $5 \mathrm{kOe}, 13 \mathrm{~K}$ at $10 \mathrm{kOe}$, and $10.5 \mathrm{~K}$ at $14 \mathrm{kOe}$ (Figure 4b). Above $18 \mathrm{kOe}$, sharp peaks at $T_{\mathrm{N} 2}$ disappeared; and above about $26 \mathrm{kOe}$, the $\chi$ versus $T$ curves became featureless. We note the presence of a kink on the $d(\chi T) / d T$ versus $T$ curves at $19 \mathrm{~K}$ at $22 \mathrm{kOe}$, which 
was still visible at $20.5 \mathrm{~K}$ at $9 \mathrm{kOe}$ (Figure S1b). Therefore, $\mathrm{BaDyFeO}_{4}$ should show a complex temperature-magnetic-field phase diagram in comparison with $\mathrm{BaYFeO}_{4}$. However, the construction of such a phase diagram is out of the scope of the present work, and single crystals are desirable for the construction of a precise phase diagram.

Isothermal magnetization curves ( $\mathrm{M}$ versus $\mathrm{H}$ ) of $\mathrm{BaDyFeO}_{4}$ at 5,15 , and $30 \mathrm{~K}$ are shown on Figure 5. The $\mathrm{M}$ versus $\mathrm{H}$ curves at $1.8 \mathrm{~K}$ and $5 \mathrm{~K}$ were very similar to each other (Figure S2). However, the $\mathrm{dM} / \mathrm{dH}$ versus $\mathrm{H}$ curve at $1.8 \mathrm{~K}$ (inset of Figure 5) shows more clearly the presence of three field-induced transitions at 6, 18, and 28 kOe. The weak transition at $6 \mathrm{kOe}$ matches well with a field-induced transition in $\mathrm{DyFeO}_{3}$ and could be caused by this impurity [20]. Moreover, the field-induced transition at $6 \mathrm{kOe}$ disappeared at $T=5 \mathrm{~K}$ above $T_{\mathrm{N}, \mathrm{Dy}}=4 \mathrm{~K}$ of $\mathrm{DyFeO}_{3}$ (Figure S2). Much stronger transitions at 18 and 28 kOe should originate from the main $\mathrm{BaDyFeO}_{4}$ phase, and these values coincide with the field values where the $\chi$ versus $T$ curves qualitatively changed (Figure $4 \mathrm{~b}$ ). The $\mathrm{M}$ versus $\mathrm{H}$ curve of $\mathrm{BaYFeO}_{4}$ at $5 \mathrm{~K}$ demonstrated a small hysteresis near the origin, which could originate from $\mathrm{YFeO}_{3}$ impurity (Figure S2), without any field-induced transitions. Therefore, the presence of the magnetic $D y^{3+}$ sublattice noticeably enriches magnetic behaviors.

Spin-glass-like magnetic properties below $17 \mathrm{~K}$ and magnetization relaxation reaching about $35 \%$ at $T=9 \mathrm{~K}$ and $\mathrm{H}=0$ Oe were observed in $\mathrm{BaYFeO}_{4}$ [9]. However, we emphasize that magnetization relaxation was only measured between 2 and $9 \mathrm{~K}$, and no results were reported above $9 \mathrm{~K}$ [9]. We also observed magnetization relaxation in our $\mathrm{BaYFeO}_{4}$ sample, but the magnetization relaxation reached maximum $3 \%$ under $\mathrm{H}=1 \mathrm{kOe}$ (Figure S1c). In addition, the relaxation was observed at all temperatures between 5 and 50 $\mathrm{K}$, that is, above $T_{\mathrm{N} 1}$. Therefore, we conclude that the magnetization relaxation in our $\mathrm{BaYFeO}_{4}$ sample is an extrinsic property. No magnetization relaxation was observed in $\mathrm{BaDyFeO}_{4}$ at 5, 10, and $20 \mathrm{~K}$ (Figure S1c).

Specific heat data of $\mathrm{BaYFeO}_{4}$ and $\mathrm{BaDyFeO}_{4}$ are shown on Figure 6. No anomalies were observed in $\mathrm{BaYFeO}_{4}$ in agreement with one of the previous reports [4]. Surprisingly, a magnetic field of $90 \mathrm{kOe}$ had no effects on specific heat data of $\mathrm{BaYFeO}_{4}$ suggesting that AFM states of $\mathrm{BaYFeO}_{4}$ are very robust. On the other hand, a very small specific heat anomaly was observed at $T_{\mathrm{N} 2}$ in $\mathrm{BaDyFeO}_{4}$, no anomalies at $T_{\mathrm{N} 1}$, and a very strong and 
broadened anomaly at $T_{\mathrm{N} 3}$ (an anomaly was also observed at $T_{\mathrm{N}, \mathrm{Dy}}=4 \mathrm{~K}$ from the $\mathrm{Dy}^{3+}$ ordering transition in $\mathrm{DyFeO}_{3}$ impurity). Therefore, the entropy release at the $\mathrm{Fe}^{3+}$ ordering transitions was also very small in $\mathrm{BaDyFeO}_{4}$ similar to $\mathrm{BaYFeO}_{4}$. A magnetic field of 90 kOe showed its effect on specific heat of $\mathrm{BaDyFeO}_{4}$ below a very high temperature of about $130 \mathrm{~K}$, far above $T_{\mathrm{N} 1}$. This fact could indicate that a magnetic field strongly polarizes the $\mathrm{Dy}^{3+}$ sublattice far above $T_{\mathrm{N} 1}$. A magnetic field of $90 \mathrm{kOe}$ completely suppressed the anomaly at $T_{\mathrm{N} 3}$. The broadened anomaly at $T_{\mathrm{N} 3}$ and its suppression by a magnetic field indicate that it is caused by $\mathrm{Dy}^{3+}$ ordering.

Frequency-dependent dielectric data of $\mathrm{BaDyFeO}_{4}$ are presented on Figure $7 \mathrm{a}$ and Figures S3-S6. Dielectric constant starts increasing below about $50 \mathrm{~K}$, which is close to $T_{\mathrm{N} 1}$ $=47 \mathrm{~K}$, but without any clear anomalies at $T_{\mathrm{N} 1}$, and it shows maxima near $35 \mathrm{~K}$. Then, dielectric constant decreases below $35 \mathrm{~K}$, and a broad peak suddenly disappears at $T_{\mathrm{N} 2}=23$ $\mathrm{K}$. There are very small dielectric peaks at $T_{\mathrm{N} 2}$ (which look frequency-independent), especially on heating curves (Figures 7a and 8a). The peak positions near $35 \mathrm{~K}$ are slightly frequency-dependent. Dielectric loss also demonstrates peaks spanning from 23 to $38 \mathrm{~K}$ (Figure 7b). Therefore, broad dielectric anomalies are present in a magnetic phase between $T_{\mathrm{N} 2}$ and $T_{\mathrm{N} 1}$ with clear hysteresis on cooling and heating at $\mathrm{H}=0$ Oe, suggesting a first-order phase transition at $T_{\mathrm{N} 2}$. Magnetic fields have noticeable effects on temperature dependence of dielectric constant (Figure 8a) resulting in the observation of positive and negative magnetodielectric effects (Figure 8b). For example, broad dielectric anomalies move to lower temperatures at $\mathrm{H}=10 \mathrm{kOe}$, especially from the low temperature side in agreement with the magnetic susceptibility data (Figure $4 \mathrm{~b}$ ) and the movement of $T_{\mathrm{N} 2}$ by magnetic fields. In other words, broad dielectric anomalies span again between $T_{\mathrm{N} 2}$ and $T_{\mathrm{N} 1}$ at $\mathrm{H}=10$ kOe with hysteresis on cooling and heating. On the other hand, no dielectric anomalies were observed in $\mathrm{BaYFeO}_{4}$ (Figure 9a), and the magnetodielectric effect was negligible (Figure $9 b)$. Therefore, the presence of the magnetic $\mathrm{Dy}^{3+}$ sublattice noticeably enriches dielectric behaviors of $\mathrm{BaDyFeO}_{4}$ in comparison with those of $\mathrm{BaYFeO}_{4}$.

Temperature dependence of dielectric constant of $\mathrm{BaYFeO}_{4}$ was qualitatively similar with that of Ref. 8, including the observation of a small kink near $25 \mathrm{~K}$ (Figure 9a). However, the absolute values were different probably because of uncertainties in pellet and 
electrode dimensions. Therefore, dielectric properties of $\mathrm{BaYFeO}_{4}$ are not so sample sensitive in comparison with the $\chi$ versus $T$ curves. In Ref. 8, difference in dielectric constant of the order of $2 \times 10^{-3}$ at $T_{\mathrm{N} 2}=35 \mathrm{~K}$ was found between the curves measured at $\mathrm{H}=0$ and 70 kOe, while no dielectric constant anomalies were detected at $\mathrm{H}=70 \mathrm{kOe}$. Our measurements were less sensitive; this could be a reason why we did not observe any dielectric anomalies in our $\mathrm{BaYFeO}_{4}$ sample at $T_{\mathrm{N} 2}=35 \mathrm{~K}$ and $\mathrm{H}=0$ Oe and other magnetic fields.

We did not observe any sharp pyroelectric current anomalies in $\mathrm{BaDyFeO}_{4}$ and $\mathrm{BaYFeO}_{4}$ (Figures 10 and 11 and Figure S8), which could be assigned to a ferroelectric transition. There were some very broad anomalies, and they reproduced very well during the change of the sign of the poling field. Therefore, they were not caused by the appearance of ferroelectric polarization. The bias electric field measurements, which should detect peaks at every ferroelectric transitions (even if a ferroelectric phase appears in intermediate temperature ranges), also did not show any peaks (Figure S9). Therefore, we conclude that dielectric anomalies in $\mathrm{BaDyFeO}_{4}$ are not caused by spin-induced ferroelectricity or spininduced ferroelectric polarization is below the detection limit of our measurements. In case of $\mathrm{BaYFeO}_{4}$, polarization of about $2 \mu \mathrm{C} / \mathrm{m}^{2}$ was measured under a poling field of $800 \mathrm{kV} / \mathrm{m}$ [8]. We could apply only $235 \mathrm{kV} / \mathrm{m}$ to our $\mathrm{BaYFeO}_{4}$ sample. If the electric polarization is a linear function of an applied electric field, we could miss polarization of about $0.6 \mu \mathrm{C} / \mathrm{m}^{2}$, which is near the detection limit. Therefore, the origin of broad dielectric peaks observed between $T_{\mathrm{N} 2}$ and $T_{\mathrm{N} 1}$ and centred near $35 \mathrm{~K}$ (at $\mathrm{H}=0$ Oe) in $\mathrm{BaDyFeO}_{4}$ remains unknown at the moment. Additional experiments will be required to understand their origin, for example, measurements using single crystals and magnetic structure determinations. We emphasize that dielectric peak positions sometimes do not match precisely with $T_{\mathrm{N}}$, for example, in o$\mathrm{LuMnO}_{3}$ [22]. Dielectric peaks near $T_{\mathrm{N}}$ and magnetodielectric effects were observed in $\mathrm{Sc}_{2} \mathrm{NiMnO}_{6}$, where no pyroelectric current anomalies were also detected [23]. Therefore, it was suggested that an antiferroelectric transition takes place in $\mathrm{Sc}_{2} \mathrm{NiMnO}_{6}$. The similar picture could be realized in $\mathrm{BaDyFeO}_{4}$.

\section{Conclusion}


We prepared a new $\mathrm{BaDyFeO}_{4}$ ferrite by a conventional solid-state method in air and investigated its structural, magnetic, and dielectric properties. Three temperature-driven magnetic transitions and two magnetic-field-driven transitions were found in $\mathrm{BaDyFeO}_{4}$. This compound shows peculiar temperature-dependent and field-dependent dielectric constant and magnetodielectric effects in comparison with $\mathrm{BaYFeO}_{4}$. However, no pyroelectric current anomalies were found suggesting the absence of spin-induced ferroelectric polarization within the sensitivity of our methods and the used pyroelectric current measurement conditions. The observation of dielectric anomalies in magnetic phases without the appearance of ferroelectric polarization is intriguing and will require further clarification.

\section{Acknowledgements}

This study was supported in part by JSPS KAKENHI Grant Number JP16H04501, a research grant from Nippon Sheet Glass Foundation for Materials Science and Engineering (40-37), Innovative Science and Technology Initiative for Security, ATLA, Japan, and the Russian Science Foundation (grant No. 19-73-10034). The synchrotron radiation experiments were performed at the SPring-8 with the approval of NIMS Synchrotron X-ray Station (Proposal Number: 2017B4502).

\section{Appendix A. Supplementary data}

Supplementary data to this paper contain details of magnetic and dielectric properties of $\mathrm{BaDyFeO}_{4}$ and $\mathrm{BaYFeO}_{4}$ and can be found online. CCDC 1899326 contains the supplementary crystallographic data for this paper. These data can be obtained free of charge via www.ccdc.cam.ac.uk/data_request/cif, or by emailing data_request@ccdc.cam.ac.uk, or by contacting The Cambridge Crystallographic Data Centre, 12 Union Road, Cambridge CB21EZ, UK; fax: +44 1223336033. 


\section{References}

[1] Kimura, T.; Goto, T.; Shintani, H.; Ishizaka, K.; Arima, T.; Tokura, Y. Magnetic Control of Ferroelectric Polarization. Nature (London) 2003, 426, 55-58.

[2] Hur, N.; Park, S.; Sharma, P. A.; Ahn, J. S.; Guha, S.; Cheong, S. W. Electric Polarization Reversal and Memory in a Multiferroic Material Induced by Magnetic Fields. Nature (London) 2004, 429, 392-395.

[3] Cheong, S. W.; Mostovoy, M. Multiferroics: a Magnetic Twist for Ferroelectricity. Nat. Mater. 2007, 6, 13-20

[4] Wrobel, F.; Kemei, M. C.; Derakhshan, S. Antiferromagnetic Spin Correlations Between Corner-Shared $\left[\mathrm{FeO}_{5}\right]^{7-}$ and $\left[\mathrm{FeO}_{6}\right]^{9-}$ Units, in the Novel Iron-Based Compound: $\mathrm{BaYFeO}_{4}$, Inorg. Chem. 2013, 52, 2671-2677.

[5] Thompson, C. M.; Greedan, J. E.; Garlea, V. O.; Flacau, R.; Tan, M.; Nguyen, P.-H. T.; Wrobel, F.; Derakhshan, S. Partial Spin Ordering and Complex Magnetic Structure in $\mathrm{BaYFeO}_{4}$ : A Neutron Diffraction and High Temperature Susceptibility Study. Inorg. Chem. 2014, 53, 1122-1127.

[6] Kato, K.; Kosuda, K.; Uchida, Y.; Takayama-Muromachi, E.; Fukunaga, O. Die Struktur des Dibariumkupferplatindiyttriumoktaoxids, $\mathrm{Ba}_{2} \mathrm{CuPtY}_{2} \mathrm{O}_{8}$. Acta Crystallogr., Sect. C: Cryst. Str. Commun. 1988, 44, 794-796.

[7] Gordon, E. E.; Derakhshan, S.; Thompson, C. M.; Whangbo, M.-H. Spin-Density Wave as a Superposition of Two Magnetic States of Opposite Chirality and Its Implications, Inorg. Chem. 2018, 57, 9782-9785.

[8] Cong, J.-Z.; Shen, S.-P.; Chai, Y.-S.; Yan, L.-Q.; Shang, D.-S.; Wang, S.-G.; Sun, Y. Spin-driven Multiferroics in $\mathrm{BaYFeO}_{4}$, J. Appl. Phys. 2015, 117, 174102.

[9] Ghara, S.; Sundaresan, A. Coexistence of Long-range Cycloidal Order and Spincluster Glass State in the Multiferroic $\mathrm{BaYFeO}_{4}$, J. Phys.: Condens. Matter. 2018, 30, 245802.

[10] Duffort, V.; Caignaert, V.; Pralong, V.; Cervellino, A.; Sheptyakov, D.; Raveau, B. Rich Crystal Chemistry and Magnetism of 114 Stoichiometric $\mathrm{LnBaFe}_{4} \mathrm{O}_{7.0}$ Ferrites. Inorg. Chem. 2013, 52, 10438-10448.

[11] Urusova, A. S.; Bryuzgina, A. V.; Mychinko, M. Yu.; Mordvinova, N. E.; Lebedev, O. I.; Caignaert, V.; Kiselev, E. A.; Aksenova, T. V.; Cherepanov, V. A. Phase Equilibria in the Y-Ba-Fe-O System, J. Alloys Compnd. 2017, 694, 375-382.

[12] Karen, P. Chemistry and Thermodynamics of the Twin Charge-ordering Transitions in $\mathrm{RBaFe}_{2} \mathrm{O}_{5+w}$ Series. J. Solid State Chem. 2004, 177, 281-292.

[13] Karen, P.; Kjekshus, A.; Huang, Q.; Lynn, J. W.; Rosov, N.; Sora, I. N.; Karen, V. L.; Mighell, A. D.; Santaro, A. Neutron and X-ray Powder Diffraction Study of $\mathrm{RBa}_{2} \mathrm{Fe}_{3} \mathrm{O}_{8+\mathrm{w}}$ Phases. J. Solid State Chem. 1998, 136, 21-33.

[14] Vallino, M.; Abbattista, F.; Mazza, D.; Delunas, A. Preparation and Characterization of $\mathrm{BaLaFeO}_{4}$. Mater. Res. Bull. 1986, 21, 733-738.

[15] Izumi, F.; Ikeda, T. A Rietveld-Analysis Program RIETAN-98 and its Applications to Zeolites. Mater. Sci. Forum 2000, 321-324, 198-205. 
[16] Tanaka, M.; Katsuya, Y.; Yamamoto, A. A New Large Radius Imaging Plate Camera for High-Resolution and High-Throughput Synchrotron X-Ray Powder Diffraction by Multiexposure Method. Rev. Sci. Instrum. 2008, 79, 075106.

[17] Tanaka, M.; Katsuya, Y.; Matsushita, Y.; Sakata, O. Development of a Synchrotron Powder Diffractometer with a One-Dimensional X-Ray Detector for Analysis of Advanced Materials. J. Ceram. Soc. Jpn. 2013, 121, 287-290.

[18] Brese, N. E.; O’Keeffe, M. Bond-Valence Parameters for Solids. Acta Crystallogr., Sect. B: Struct. Sci. 1991, 47, 192-197.

[19] Shang, M.; Zhang, C. Y.; Zhang, T. S.; Yuan, L.; Ge, L.; Yuan, H. M.; Feng, S. H. The Multiferroic Perovskite $\mathrm{YFeO}_{3}$. Appl. Phys. Lett. 2013, 102, 062903.

[20] Zhao, Z. Y.; Zhao, X.; Zhou, H. D.; Zhang, F. B.; Li, Q. J.; Fan, C.; Sun, X. F.; Li, X. G. Ground State and Magnetic Phase Transitions of Orthoferrite $\mathrm{DyFeO}_{3}$. Phys. Rev. B: Condens. Matter Mater. Phys. 2014, 89, 224405.

[21] Wang, J. C.; Liu, J .J.; Sheng, J .M.; Luo, W.; Ye, F.; Zhao, Z. Y.; Sun, X. F.; Danilkin, S. A.; Deng, G. C.; Bao, W. Simultaneous Occurrence of Multiferroism and Short-Range Magnetic Order in $\mathrm{DyFeO}_{3}$. Phys. Rev. B: Condens. Matter Mater. Phys. 2016, 93, 140403.

[22] Zhang, L.; Dönni, A.; Pomjakushin, V. Y.; Yamaura, K.; Belik A. A. Crystal and Magnetic Structures and Properties of $\left(\mathrm{Lu}_{1-\mathrm{x}} \mathrm{Mn}_{\mathrm{x}}\right) \mathrm{MnO}_{3}$ Solid Solutions. Inorg. Chem. 2018, 57, 14073-14085.

[23] Yi, W.; Princep, A. J.; Guo, Y. F.; Johnson, R. D.; Khalyavin, D.; Manuel, P.; Senyshyn, A.; Presniakov, I. A.; Sobolev, A. V.; Matsushita, Y.; Tanaka, M.; Belik, A. A.; Boothroyd, A. T. $\mathrm{Sc}_{2} \mathrm{NiMnO}_{6}$ : A Double-Perovskite with a Magnetodielectric Response Driven by Multiple Magnetic Orders. Inorg. Chem. 2015, 54, 8012-8021. 


\section{Table 1}

Structure parameters of $\mathrm{BaDyFeO}_{4}$ at $295 \mathrm{~K}$ from synchrotron X-ray powder diffraction data.

\begin{tabular}{|c|c|c|c|c|c|}
\hline Site & WP & $x$ & $y$ & Z & $B\left(\AA^{2}\right)$ \\
\hline Ba1 & $4 c$ & $0.21160(4)$ & 0.25 & $0.67406(5)$ & $0.715(9)$ \\
\hline $\mathrm{Ba} 2$ & $4 c$ & $0.41492(4)$ & 0.25 & $0.39532(4)$ & $0.760(8)$ \\
\hline Dy1 & $4 c$ & $0.41447(3)$ & 0.25 & $0.01463(3)$ & $0.536(6)$ \\
\hline Dy2 & $4 c$ & $0.14330(3)$ & 0.25 & $0.30953(4)$ & $0.540(6)$ \\
\hline $\mathrm{Fe} 1$ & $4 c$ & $0.46928(8)$ & 0.25 & 0.71533(11) & $0.554(18)$ \\
\hline $\mathrm{Fe} 2$ & $4 c$ & $0.18974(9)$ & 0.25 & $0.02269(10)$ & $0.488(18)$ \\
\hline $\mathrm{O} 1$ & $4 c$ & $0.5870(4)$ & 0.25 & $0.6153(4)$ & $0.38(8)$ \\
\hline $\mathrm{O} 2$ & $4 c$ & $0.2927(4)$ & 0.25 & $0.1791(5)$ & $1.08(10)$ \\
\hline $\mathrm{O} 3$ & $8 d$ & $0.0050(3)$ & $0.5090(8)$ & $0.3591(3)$ & $1.33(8)$ \\
\hline $\mathrm{O} 4$ & $8 d$ & $0.2194(3)$ & $0.5090(7)$ & $0.4410(4)$ & $1.36(8)$ \\
\hline $\mathrm{O} 5$ & $8 d$ & $0.1113(3)$ & $0.9977(7)$ & $0.1314(3)$ & $0.81(7)$ \\
\hline
\end{tabular}

WP: Wyckoff position. The occupation factor of each site is unity. Space group Pnma (No 62); $Z=8$.

$a=13.16861(1) \AA, b=5.70950(1) \AA, c=10.26783(1) \AA$, and $V=771.9985(11)$

$\AA^{3} ; R_{\mathrm{wp}}=3.20 \%, R_{\mathrm{p}}=2.44 \%, R_{\mathrm{B}}=3.70 \%$, and $R_{\mathrm{F}}=2.22 \% ; \rho_{\text {cal }}=7.222$ $\mathrm{g} / \mathrm{cm}^{3}$. DyFeO 3 impurity: 1.9 wt. \%. 


\section{Table 2}

Selected bond lengths $(l(\AA)<3.25 \AA \text { ) and bond valence sums (BVS) })^{\mathrm{a}}$ in $\mathrm{BaDyFeO}_{4}$ at $295 \mathrm{~K}$

\begin{tabular}{cccc}
\hline Ba1 - O1 & $2.715(5)$ & $\mathrm{Ba} 2-\mathrm{O} 2$ & $2.741(5)$ \\
$\mathrm{Ba} 1-\mathrm{O} 5(\times 2)$ & $2.763(4)$ & $\mathrm{Ba} 2-\mathrm{O} 5(\times 2)$ & $2.827(4)$ \\
$\mathrm{Ba} 1-\mathrm{O} 4(\times 2)$ & $2.815(4)$ & $\mathrm{Ba} 2-\mathrm{O} 1(\times 2)$ & $2.857(4)$ \\
$\mathrm{Ba} 1-\mathrm{O} 2(\times 2)$ & $2.856(4)$ & $\mathrm{Ba} 2-\mathrm{O} 5(\times 2)$ & $2.973(4)$ \\
$\mathrm{Ba} 1-\mathrm{O} 3(\times 2)$ & $3.185(4)$ & $\mathrm{Ba}-\mathrm{O} 4(\times 2)$ & $3.006(4)$ \\
$\mathrm{Ba} 1-\mathrm{O} 4(\times 2)$ & $3.199(4)$ & $\mathrm{Ba} 2-\mathrm{O} 1$ & $3.200(5)$ \\
$\mathrm{BVS}\left(\mathrm{Ba} 1^{2+}\right)^{\mathrm{a}}$ & 2.14 & $\mathrm{Ba} 2-\mathrm{O} 3(\times 2)$ & $3.228(4)$ \\
& & & \\
$\mathrm{Dy} 1-\mathrm{O} 3(\times 2)$ & $2.300(4)$ & $\mathrm{BVS}\left(\mathrm{Ba} 2^{2+}\right)^{\mathrm{a}}$ & 2.05 \\
$\mathrm{Dy} 1-\mathrm{O} 2$ & $2.329(5)$ & $\mathrm{Dy} 2-\mathrm{O} 4(\times 2)$ & $2.239(4)$ \\
$\mathrm{Dy} 1-\mathrm{O} 3(\times 2)$ & $2.360(4)$ & $\mathrm{Dy} 2-\mathrm{O} 5(\times 2)$ & $2.367(4)$ \\
$\mathrm{Dy} 1-\mathrm{O} 4(\times 2)$ & $2.361(4)$ & $\mathrm{Dy} 2-\mathrm{O} 2$ & $2.380(5)$ \\
$\mathrm{BVS}\left(\mathrm{Dy} 1^{3+}\right)^{\mathrm{a}}$ & 3.10 & $\mathrm{Dy} 2-\mathrm{O} 3(\times 2)$ & $2.401(4)$ \\
$\mathrm{Fe} 1-\mathrm{O} 1$ & $1.860(5)$ & $\mathrm{BVS}\left(\mathrm{Dy} 2^{3+}\right)^{\mathrm{a}}$ & 3.11 \\
$\mathrm{Fe} 1-\mathrm{O} 5(\times 2)$ & $1.967(4)$ & $\mathrm{Fe} 2-\mathrm{O} 1$ & $1.958(5)$ \\
$\mathrm{Fe} 1-\mathrm{O} 3(\times 2)$ & $2.046(4)$ & $\mathrm{Fe} 2-\mathrm{O} 4(\times 2)$ & $2.007(4)$ \\
$\mathrm{BVS}\left(\mathrm{Fe} 1^{3+}\right)^{\mathrm{a}}$ & 2.82 & $\mathrm{Fe} 2-\mathrm{O} 5(\times 2)$ & $2.095(4)$ \\
& & $\mathrm{Fe} 2-\mathrm{O} 2$ & $2.103(5)$ \\
\hline & & $\mathrm{BVS}\left(\mathrm{Fe} 2^{3+}\right)^{\mathrm{a}}$ & 2.81 \\
\hline
\end{tabular}

${ }^{a} \mathrm{BVS}=\sum_{i=1}^{N} v_{i}, v_{i}=\exp \left[\left(R_{0}-l_{i}\right) / B\right], N$ is the coordination number, $B=0.37, R_{0}\left(\mathrm{Ba}^{2+}\right)=2.29$, $R_{0}\left(\mathrm{Dy}^{3+}\right)=2.036$, and $R_{0}\left(\mathrm{Fe}^{3+}\right)=1.759$ [18] 


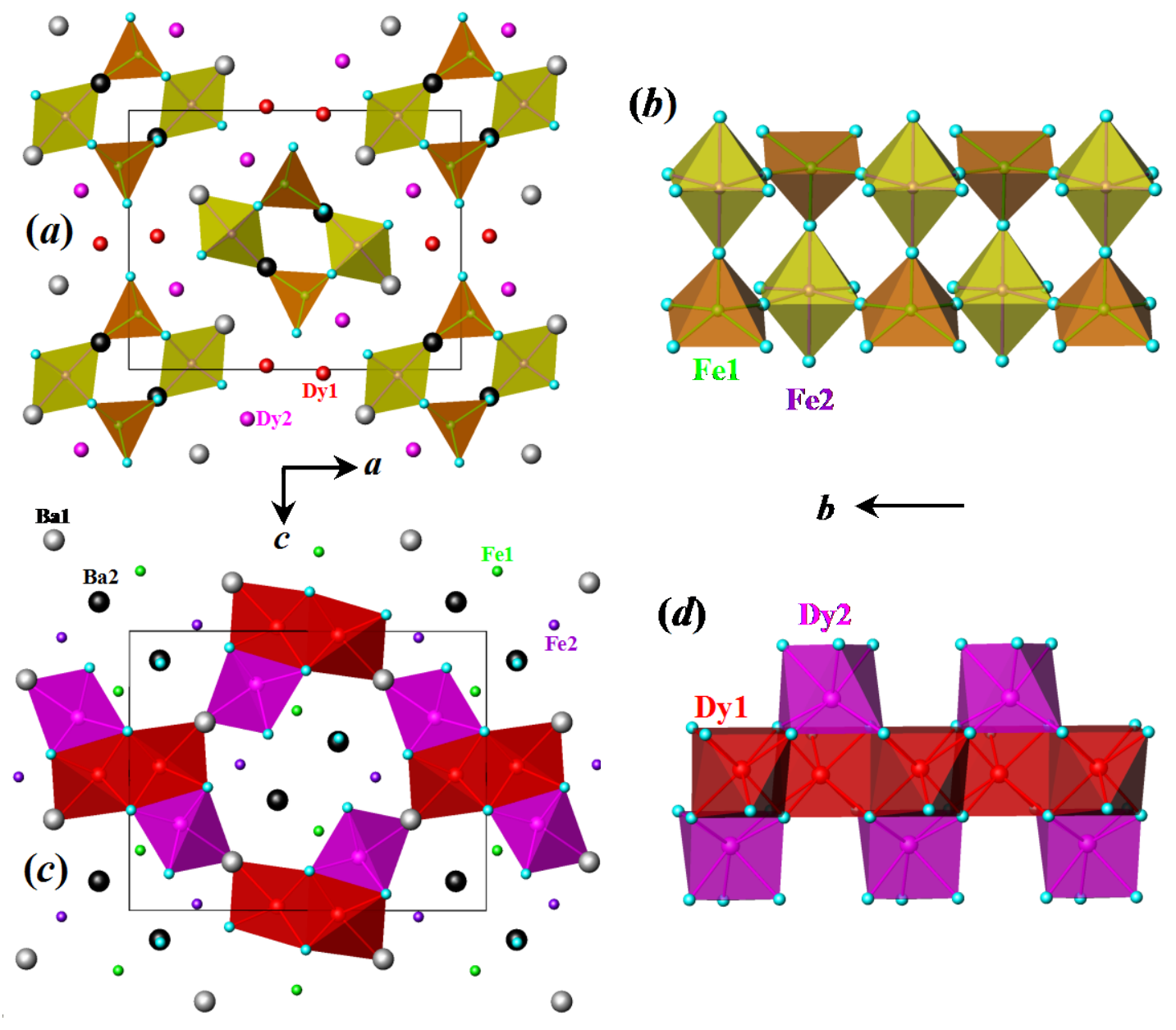

Figure 1. Crystal structure of $\mathrm{BaDyFeO}_{4}$. (a) Connection of the $\mathrm{Fe} 1 \mathrm{O}_{5}$ and $\mathrm{Fe}_{2} \mathrm{O}_{6}$ polyhedra viewed along the $b$ axis. (b) One chain of the $\mathrm{Fe} 1 \mathrm{O}_{5}$ and $\mathrm{Fe} 2 \mathrm{O}_{6}$ polyhedra along the $b$ axis. (c) Connection of the $\mathrm{Dy}_{10} \mathrm{O}_{7}$ and $\mathrm{Dy}_{2} \mathrm{O}_{7}$ polyhedra viewed along the $b$ axis. (d) One chain of the $\mathrm{Dy}_{1} \mathrm{O}_{7}$ and $\mathrm{Dy}_{2} \mathrm{O}_{7}$ polyhedra along the $b$ axis. 


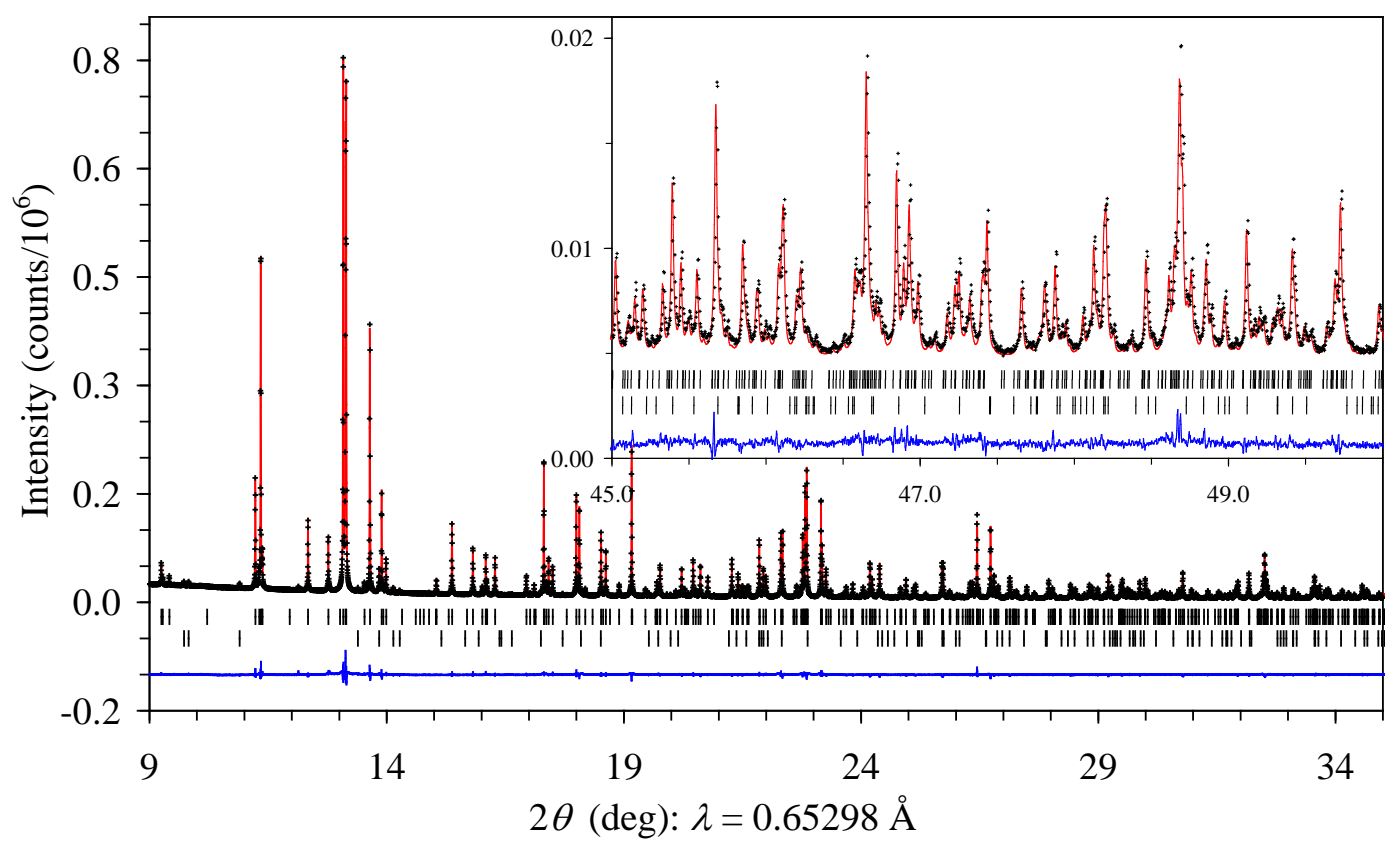

Figure 2. A fragment (9-35) of experimental (black crosses), calculated (red line), and difference (blue line) synchrotron XRPD patterns of $\mathrm{BaDyFeO}_{4}$ at $T=295 \mathrm{~K}$. The tick marks show possible Bragg reflection positions for $\mathrm{BaDyFeO}_{4}$ (the first row) and $\mathrm{DyFeO}_{3}$ impurity (the second row). The inset shows an enlarged fragment $\left(45-50^{\circ}\right)$. 


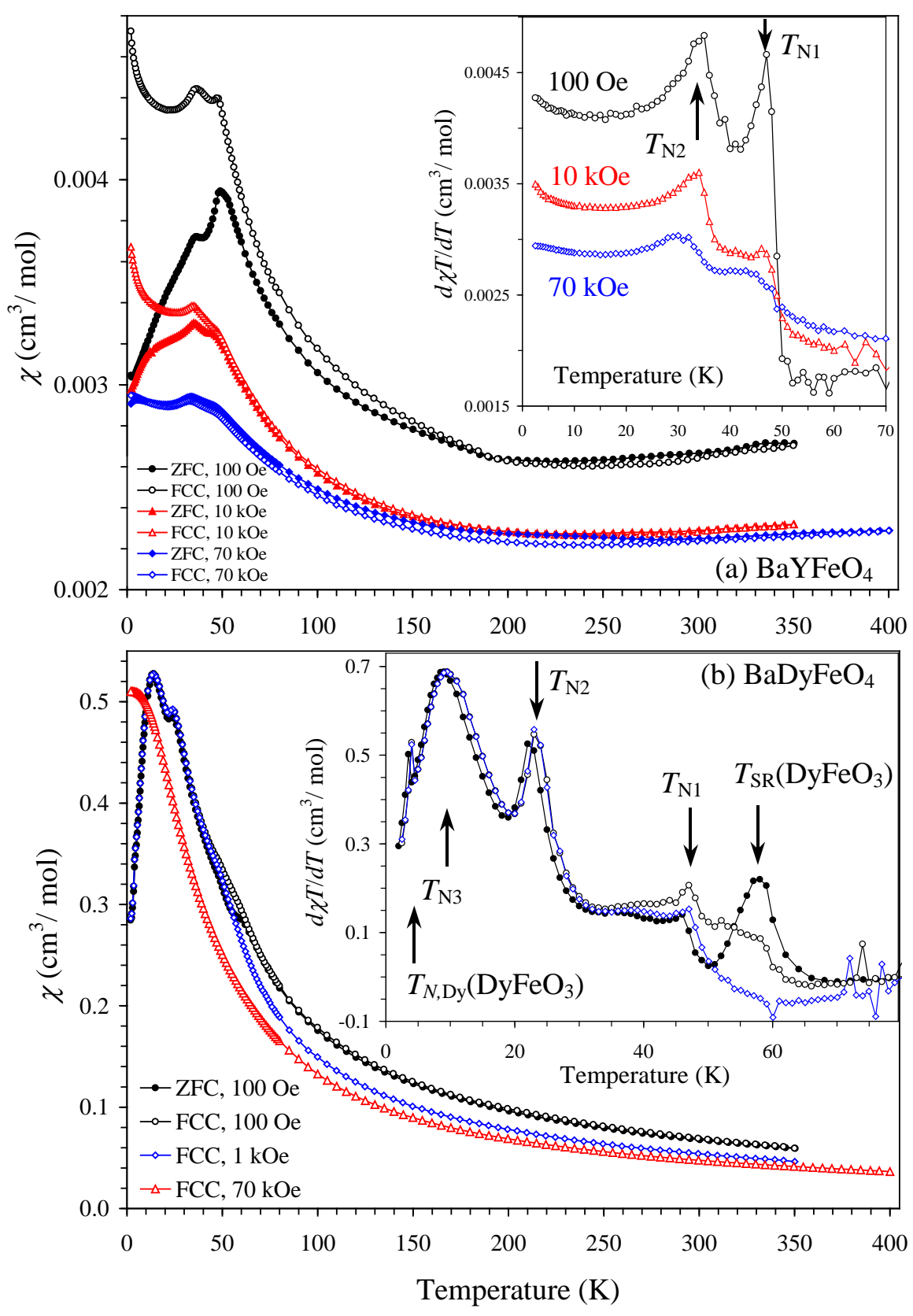

Figure 3. ZFC (filled symbols) and FCC (empty symbols) dc magnetic susceptibility ( $\chi=$ $\mathrm{M} / \mathrm{H}$ ) curves of (a) $\mathrm{BaYFeO}_{4}$ and (b) $\mathrm{BaDyFeO}_{4}$ at different magnetic fields. The insets show the $d(\chi T) / d T$ versus $T$ curves to emphasize magnetic anomalies. $T_{\mathrm{N}}$ : Néel tempereature, $T_{\mathrm{SR}}$ : spin-reorientation transition temperature. 


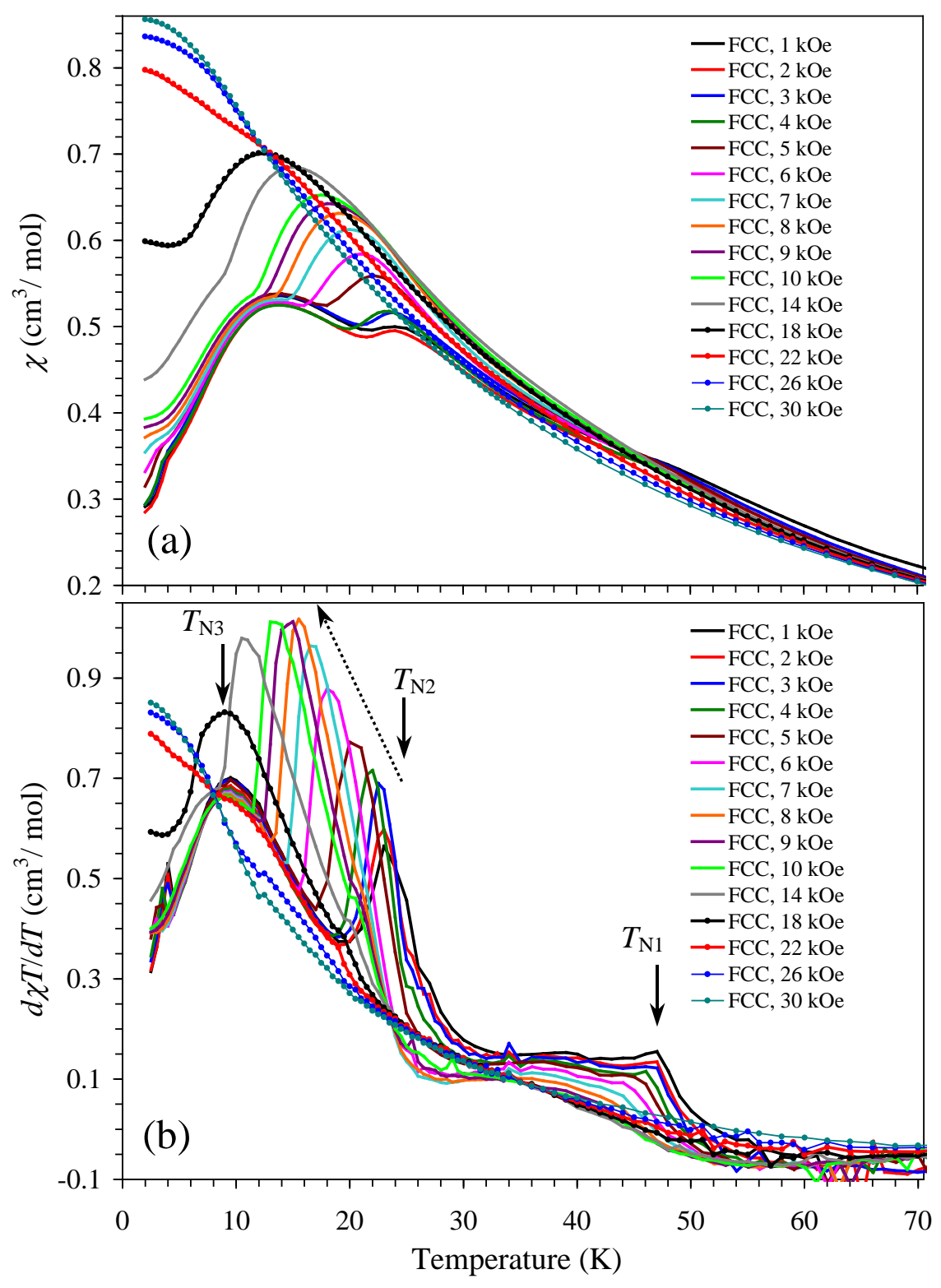

Figure 4. (a) FCC dc magnetic susceptibility $(\chi=\mathrm{M} / \mathrm{H})$ curves of $\mathrm{BaDyFeO}_{4}$ at different magnetic fields from $1 \mathrm{kOe}$ to $30 \mathrm{kOe}$. (b) $d(\chi T) / d T$ versus $T$ curves at the same magnetic fields to emphasize magnetic anomalies. 


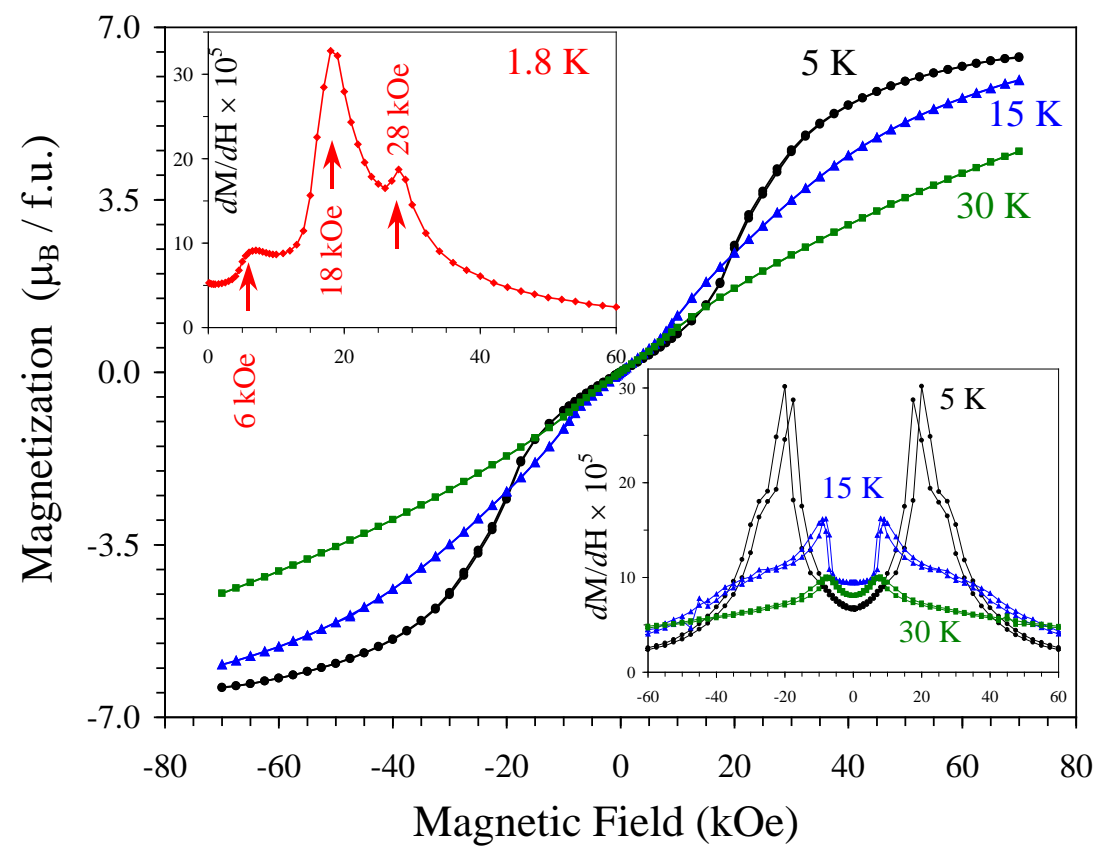

Figure 5. $\mathrm{M}$ versus $\mathrm{H}$ curves at $T=5,15$, and $30 \mathrm{~K}$ for $\mathrm{BaDyFeO}_{4}$. (f.u.: formula unit). The insets show the $d \mathrm{M} / d \mathrm{H}$ versus $\mathrm{H}$ curves to emphasize field-induced phase transitions at $T=$ $1.8 \mathrm{~K}$ (left) and $T=5,15$, and $30 \mathrm{~K}$ (right). 


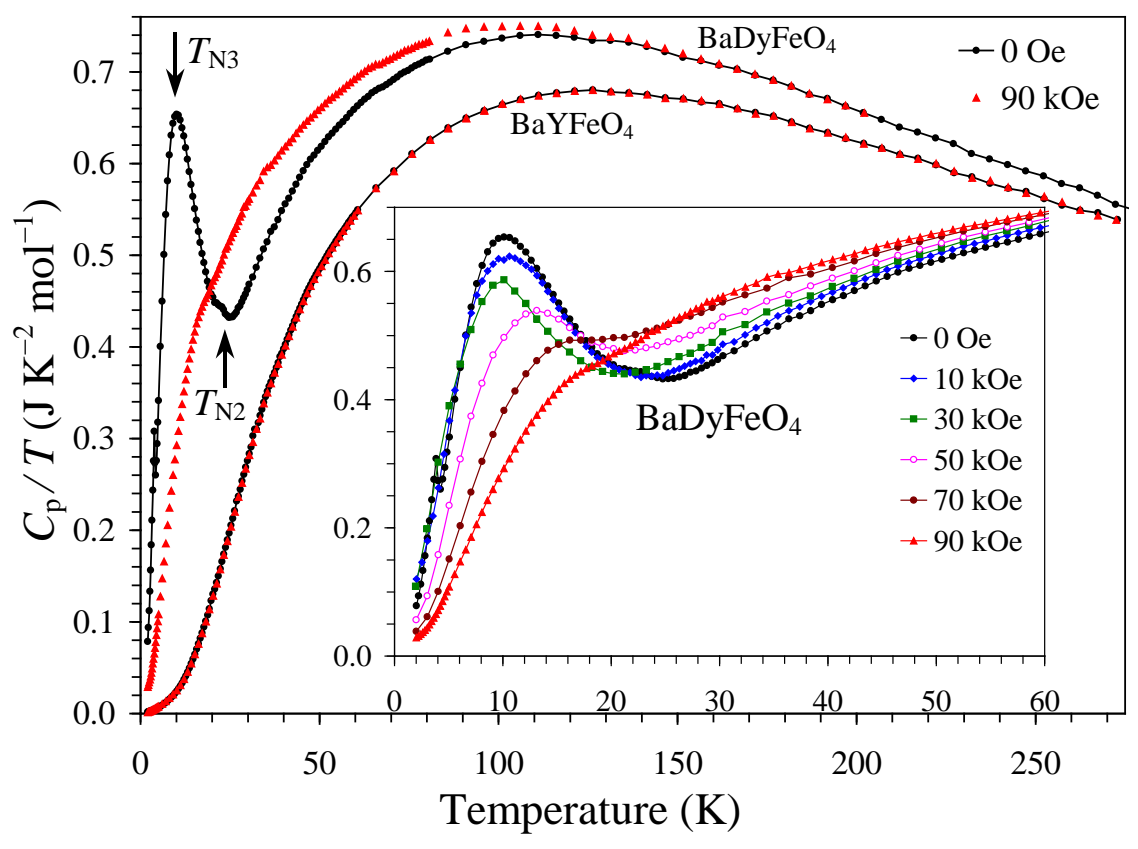

Figure 6. Specific heat, plotted as $C_{\mathrm{p}} / T$ versus $T$, of $\mathrm{BaDyFeO}_{4}$ and $\mathrm{BaYFeO}_{4}$ at $\mathrm{H}=0$ (black) and $90 \mathrm{kOe}$ (red). Measurements were performed on cooling. The inset shows $C_{\mathrm{p}} / T$ versus $T$ curves below $60 \mathrm{~K}$ at different magnetic fields (measured on cooling) for $\mathrm{BaDyFeO}_{4}$. 


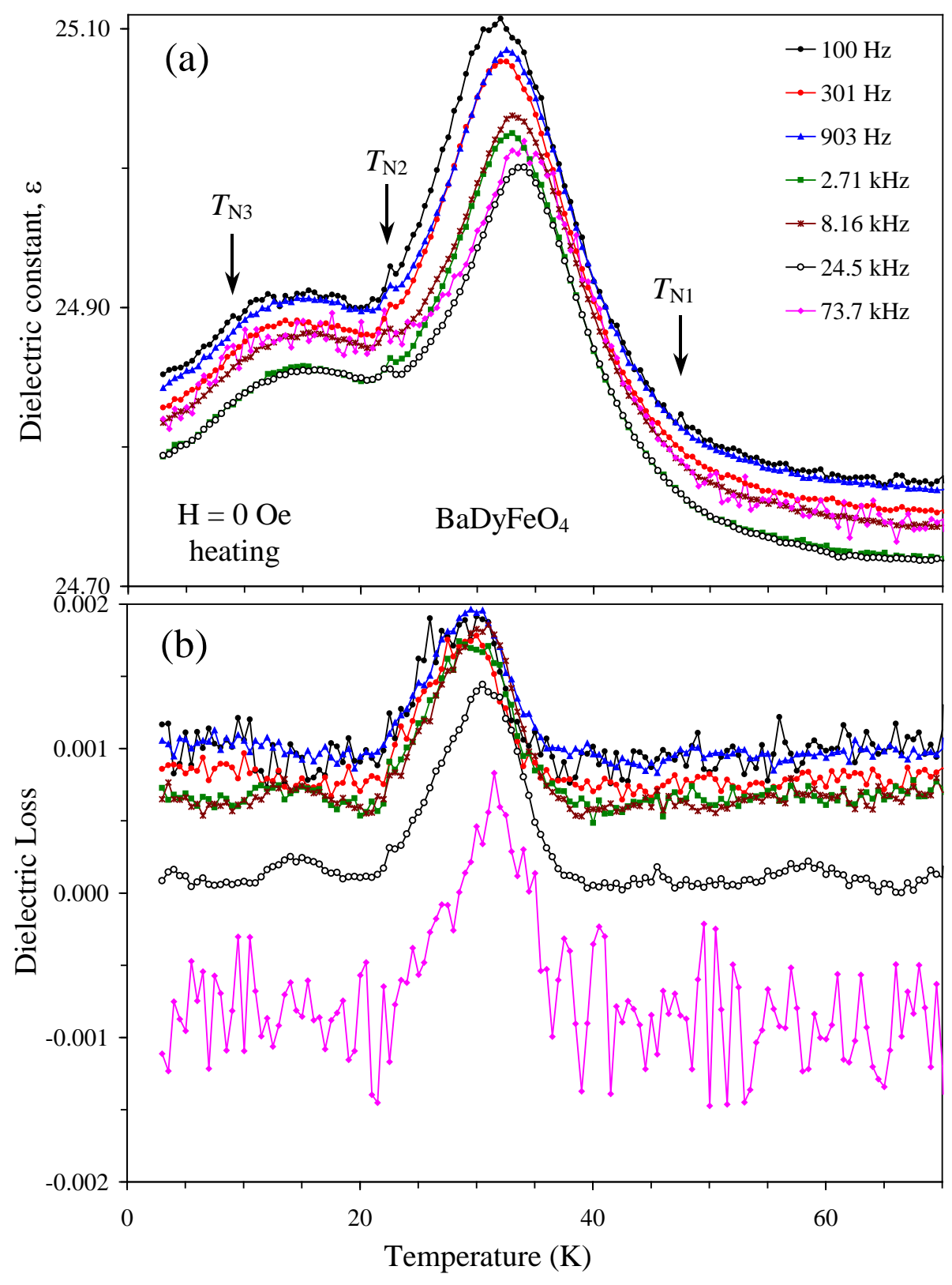

Figure 7. (a) Dielectric constant and (b) dielectric loss of $\mathrm{BaDyFeO}_{4}$ as functions of temperature. Measurements were performed on heating at zero magnetic field at different frequencies. 

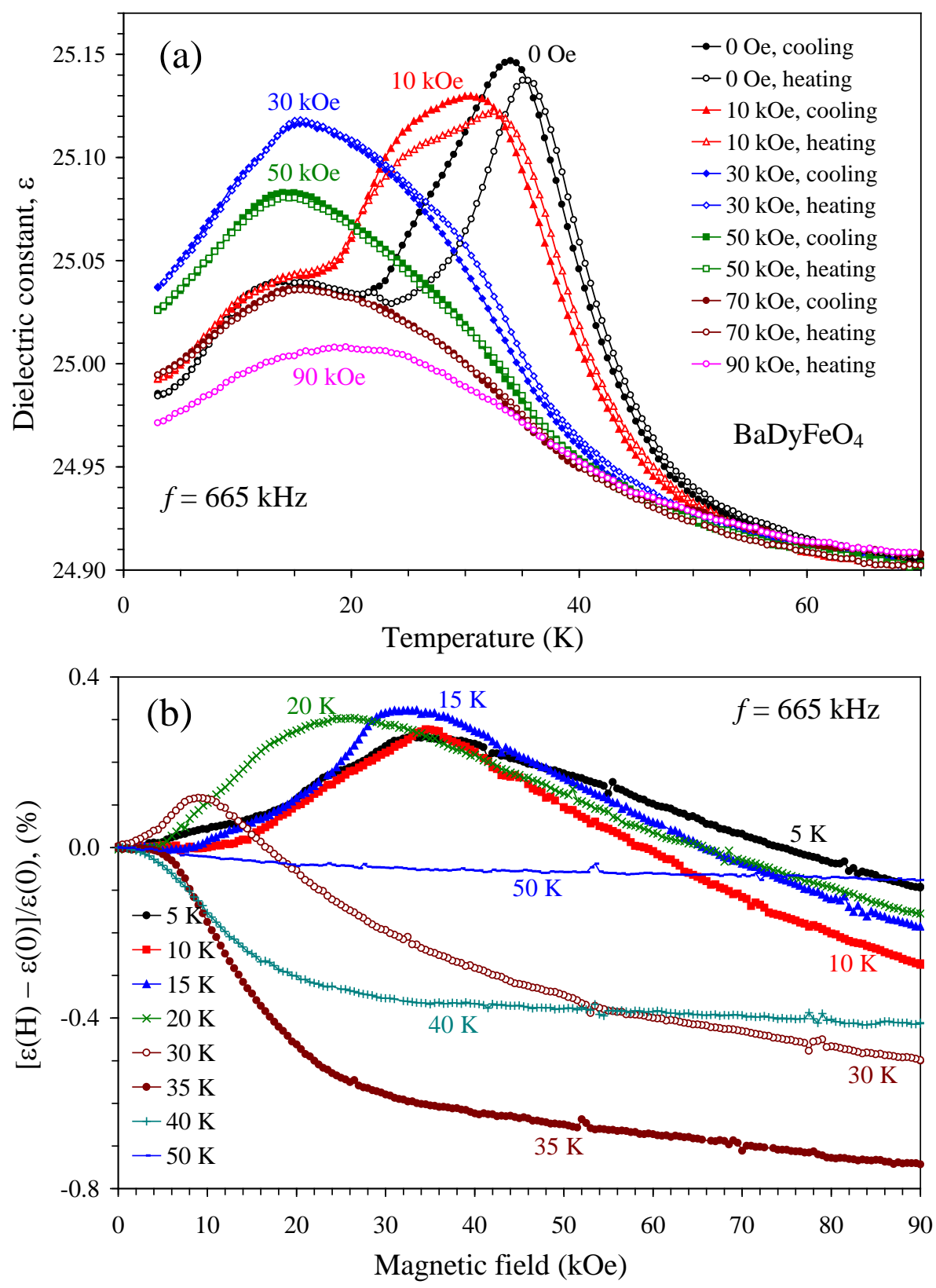

Figure 8. (a) Temperature dependence of dielectric constant in $\mathrm{BaDyFeO}_{4}$. Measurements were performed on cooling (filled symbols) and heating (empty symbols) at $\mathrm{H}=0,10,30$, 50, 70, and $90 \mathrm{kOe}$. Curves at one frequency of $665 \mathrm{kHz}$ are shown. (b) Magnetic-field dependence of relative changes of dielectric constant (magnetodielectric effects) in $\mathrm{BaDyFeO}_{4}$ at $T=5,10,15,20,30,35,40$, and $50 \mathrm{~K}$. Curves at one frequency of $665 \mathrm{kHz}$ are shown. 

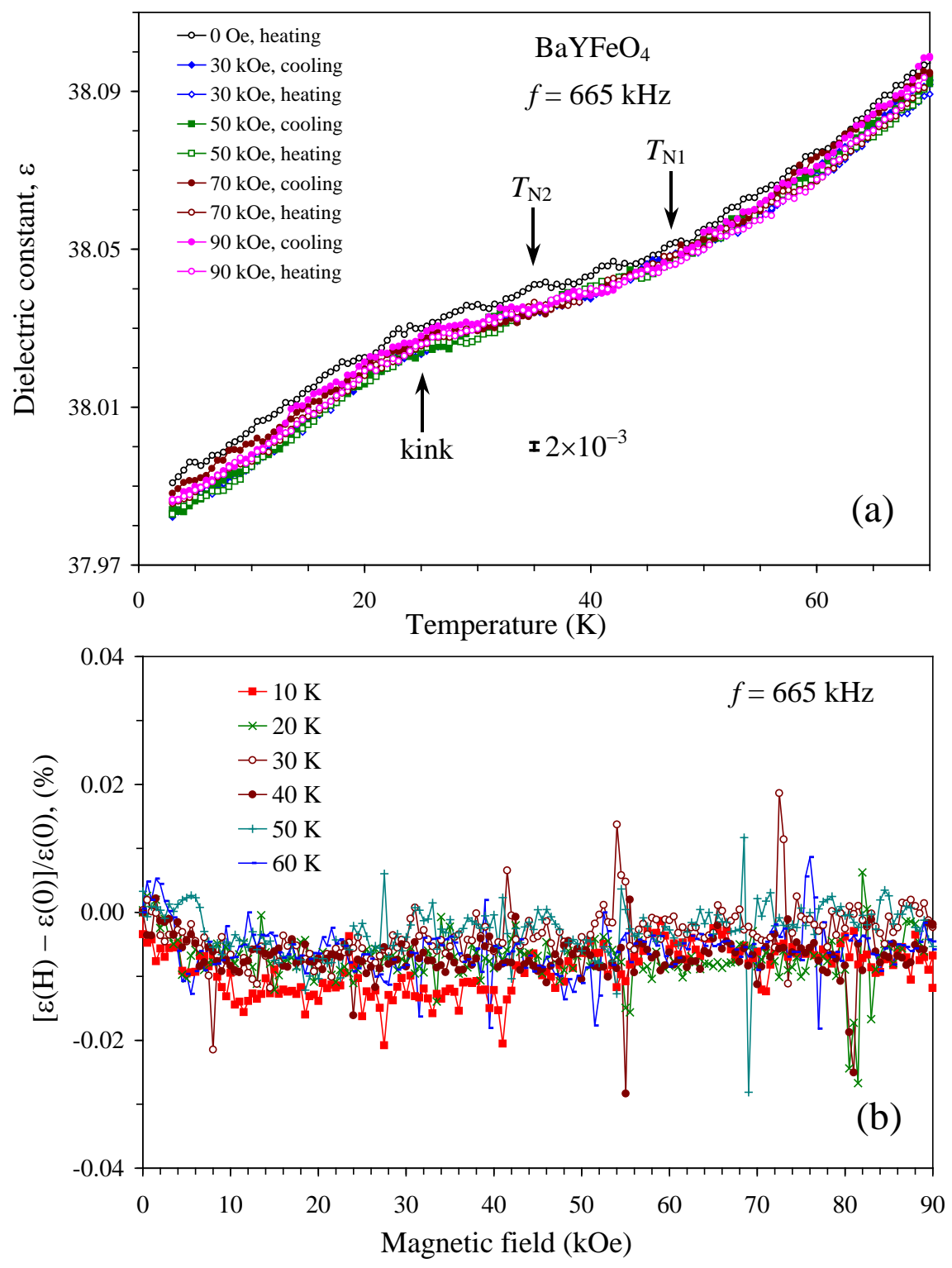

Figure 9. (a) Temperature dependence of dielectric constant in $\mathrm{BaYFeO}_{4}$. Measurements were performed on cooling (filled symbols) and heating (empty symbols) at $\mathrm{H}=0,30,50$, 70 , and $90 \mathrm{kOe}$. Curves at one frequency of $665 \mathrm{kHz}$ are shown. An error bar of $2 \times 10^{-3}$ is shown - a dielectric constant anomaly of this order was detected in Ref. [8]. (b) Magneticfield dependence of relative changes of dielectric constant (magnetodielectric effects) in $\mathrm{BaYFeO}_{4}$ at $T=10,20,30,40,50$, and $60 \mathrm{~K}$. Curves at one frequency of $665 \mathrm{kHz}$ are shown. 

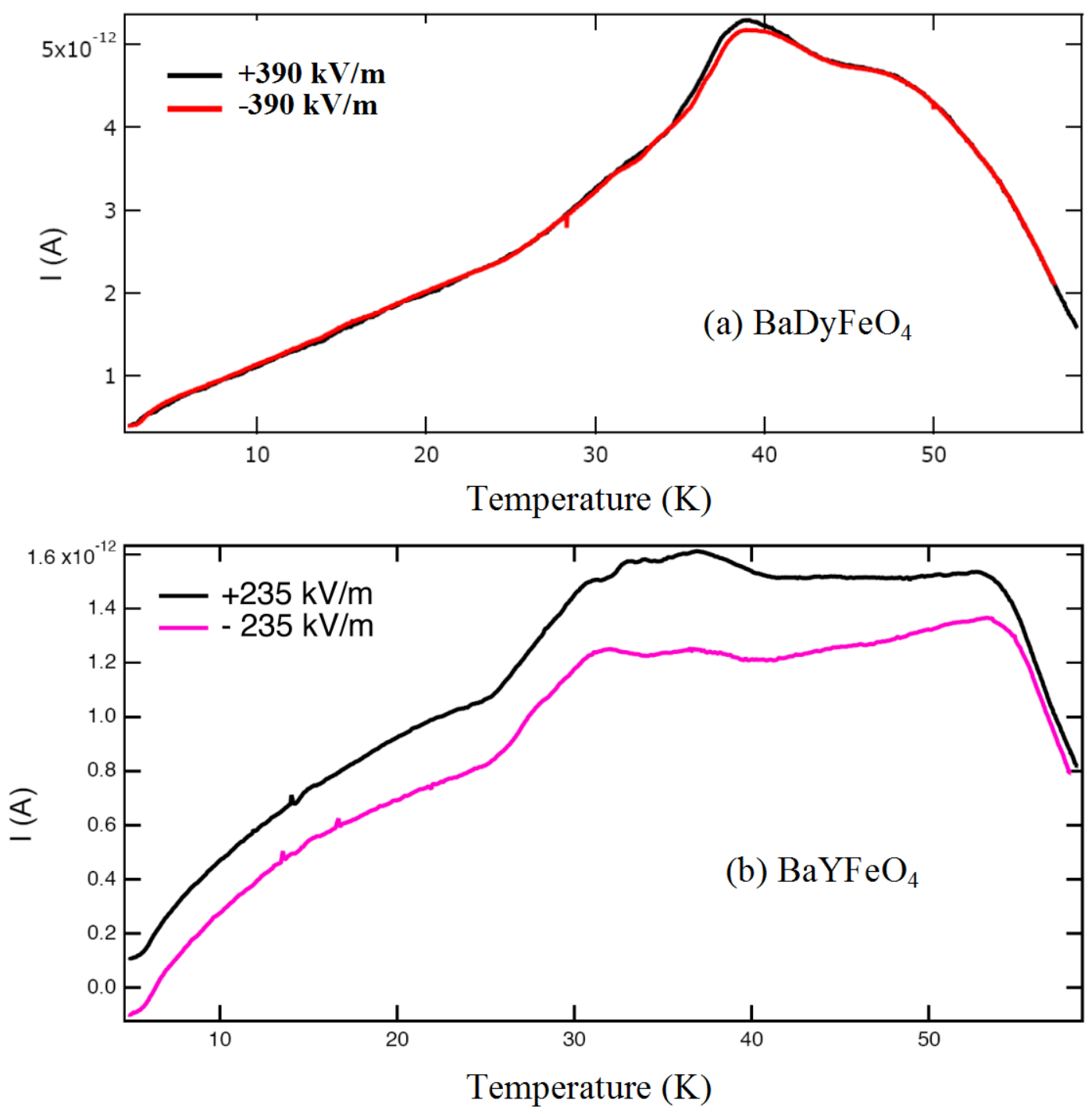

Figure 10. Results of pyroelectric current measurements in (a) $\mathrm{BaDyFeO}_{4}$ and (b) $\mathrm{BaYFeO}_{4}$. The poling was performed from $100 \mathrm{~K}$ to $2 \mathrm{~K}$ under the poling fields $E_{\mathrm{P}}=+390$ and -390 $\mathrm{kV} / \mathrm{m}$ for $\mathrm{BaDyFeO}_{4}$ and $E_{\mathrm{P}}=+235$ and $-235 \mathrm{kV} / \mathrm{m}$ for $\mathrm{BaYFeO}_{4}$ at $\mathrm{H}=0$ Oe. 


\section{Highlights:}

- $\mathrm{BaDyFeO}_{4}$ ferrite was prepared by a solid-state method.

- $\mathrm{BaDyFeO}_{4}$ shows three magnetic transitions.

- $\mathrm{BaDyFeO}_{4}$ shows two magnetic-field-induced transitions.

- $\mathrm{BaDyFeO}_{4}$ demonstrates complex behaviors of dielectric constant.

\section{Graphical Abstract}

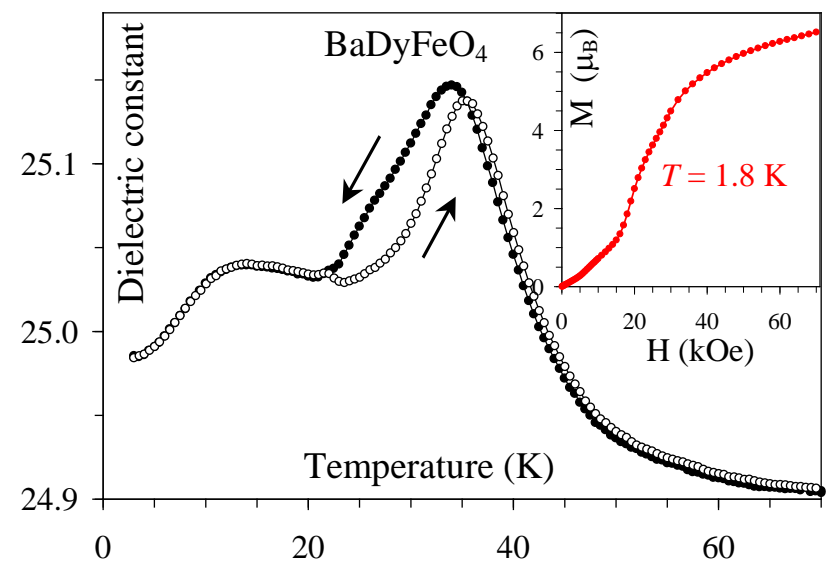

